\title{
Polarized Emission and the Discovery of New Magnetic CVs
}

\author{
Gary D. Schmidt \\ Steward Observatory, The University of Arizona, Tucson, AZ 85721 \\ USA
}

\begin{abstract}
Recent quasar surveys have identified several new magnetic cataclysmic variables accreting at remarkably low rates, $\sim 10^{-13} M_{\odot}$ $\mathrm{yr}^{-1}$. The new discoveries raise questions regarding selection effects that may influence the current sample and the traditional evolutionary conclusions that have been drawn. This paper reviews the techniques that have been used to identify polars, including a summary of the optical polarization as a function of accretion rate and magnetic field strength. The new, low- $\dot{m}$ systems accrete without a shock and cooling is dominated by optical cyclotron emission in very well-defined harmonics. These binaries, which have been found to accrete at far below the rate corresponding to angular momentum loss by gravitational radiation, also appear to contain unusually cool white dwarfs, suggesting that they are either at an advanced age or in unusual evolutionary states.
\end{abstract}

\section{Samples and Selection Effects}

Among the physical processes that are uniquely accessible in the magneticallycontrolled cataclysmic variables, or "polars", are the mechanisms that regulate accretion onto the white dwarf. With flight times from L1 measured in minutes and no disk to store the gas or dilute the accretion luminosity, the photometric record of an AM Her-type system in X-rays and optical light portrays the effects of instabilities and turbulent processes that arise en route. Brightness fluctuations have been recorded on time scales ranging from seconds to years, and explanations for various types of behavior have included such diverse influences as star spots on the secondary, instabilities at the L1 nozzle, blob fragmentation in the magnetic threading region, and shock oscillations in the accretion column.

Though amenable to diagnosing stream and shock characteristics, the lack of any time averaging within the binary makes it difficult to judge the secular accretion rate, and therefore the evolutionary status of a given system. Polars are well known to undergo high and low accretion states, between which the accretion rate may vary by 2 orders of magnitude or more. Low states may persist for weeks to many months, and in at least one case, appear to be more the rule than the exception (AR UMa; Schmidt et al. 1999). The cause(s) for these marked variations in mass transfer are not understood, and their wide variation in character from one system to another suggests that, among CVs in general, departures from secular accretion may be large and protracted. 


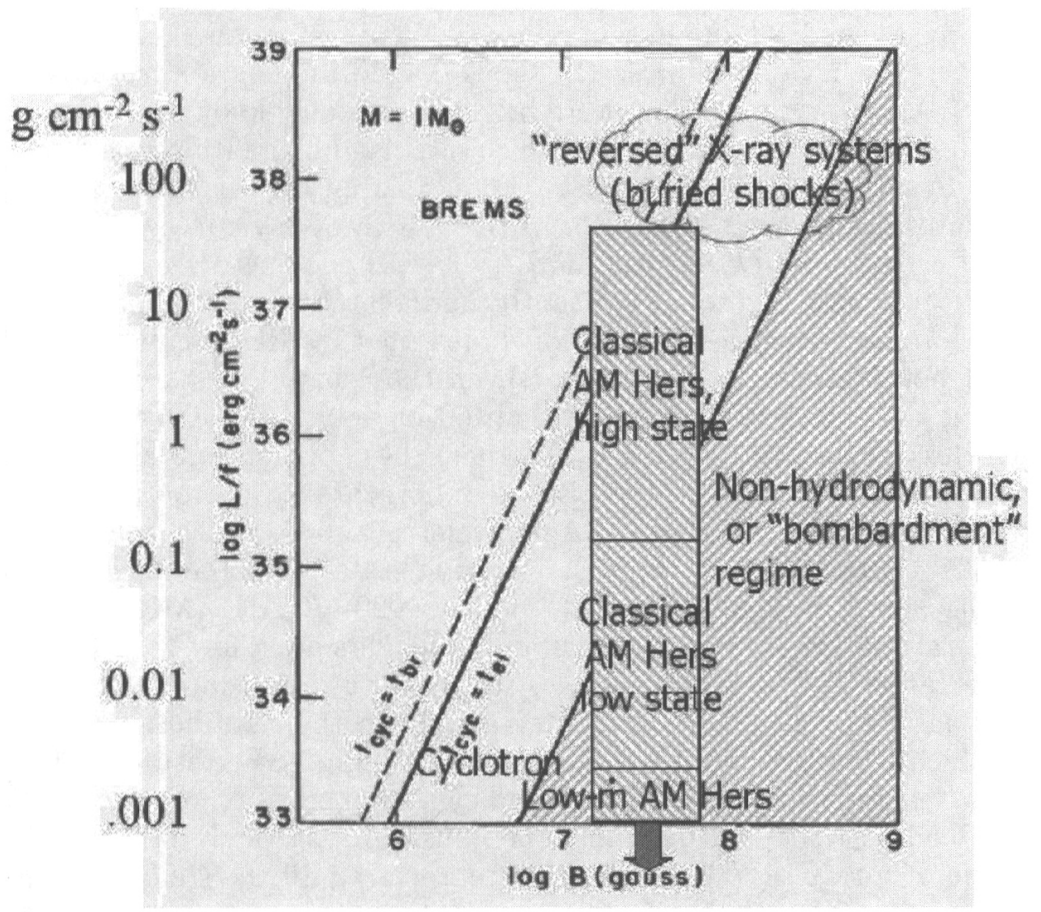

Figure 1. Primary cooling modes for accretion onto white dwarfs. Bremsstrahlung losses, which dominate at low magnetic fields and/or high specific accretion rates, give way to cyclotron emission as the primary coolant at higher fields and lower accretion rates. Approximate regions occupied by various "classes" of polar are indicated. At the very low values of $\dot{m}$ found in recent faint optical surveys, accretion moves from a shock situation into the non-hydrodynamic, or "bombardment" regime in the lower right portion of the diagram. Here, the accretion energy is deposited directly into the atmosphere, and the emission regions radiate primarily in cyclotron lines indicative of very low plasma temperatures ( $\$ 1 \mathrm{keV})$. Figure adapted from Lamb \& Masters (1979). 
These considerations are complicated by the standard selection effects that plague incomplete samples. A flux-limited survey naturally over-represents objects that are unusually luminous in the waveband being used. Referring to Figure 1, where we have reproduced the shock diagnostic diagram from Lamb \& Masters (1979), the original polars were bright, previously-known variable stars (e.g., AM Her, VV Pup, AN UMa) in which circular polarization was found to be strong. As such, they would tend to be reasonably high- $m$ systems in high accretion states. With the advent of sensitive all-sky X-ray surveys from $E I N$ $S T E I N$ and especially $R O S A T$, the hard $(\sim 2-10 \mathrm{keV}) \mathrm{X}$-ray emission from the radial shocks could be probed efficiently, and a large number of new systems were found. Indeed, Warner (1995) concluded that "...ROSAT has probably detected all polars with $m_{v}<20$, apart from those in a low state during the survey." The current catalog of more than 60 objects is heavily weighted toward such discoveries. As implied by Warner, these objects are also likely to be biased toward relatively high specific accretion rates - and particularly toward bremsstrahlung-cooled shocks. Again referring to Figure 1, such systems have $\dot{m} \sim 0.1-10 \mathrm{~g} \mathrm{~cm}^{-2} \mathrm{~s}^{-1}$ in high accretion states. For the magnetic field strengths measured in these "classical" polars, $\sim 20-70 \mathrm{MG}\left(\mathrm{MG}=10^{6} \mathrm{G}\right)$, shock temperatures are $\sim 10-20 \mathrm{keV}$, standoff heights are a few $\%$ of the white dwarf radius, and (for homogeneous flows) the cooling regions are roughly "pillbox" in shape. Sketches of this geometry appear in the rightmost columns of Figure 2, which is a reproduction from Wickramasinghe \& Ferrario (2000). Because of the elevated shock, hard X-rays and cyclotron emission can escape from the top and sides of the settling zone, the emission is strongly polarized, and the flux ratio of soft X-rays/EUV emission reprocessed off the stellar surface to bremsstrahlung is SXR/HXR $\sim 50: 50$.

\section{The Apparent Lack of High-Field Systems}

The "reversed" X-ray modes of several polars observed with EXOSAT and ROSAT (e.g., AM Her, Heise et al. 1985; UZ For, Ramsay et al. 1993), where $\mathrm{SXR} / \mathrm{HXR}$ can exceed a factor 10, revealed that under certain circumstances gas accretes in over-dense "blobs" whose local ram pressure is sufficient to depress the shock below the photosphere and thus prevent efficient energy loss in the primary shock coolants. The result is a photospheric patch strongly heated from below that emits primarily in the SXR/EUV. This geometry is indicated in the left column of sketches in Figure 2.

In retrospect, these "buried shock" systems should have been clues toward solving at least part of the puzzle of the "missing" high magnetic-field polars. Among the 60-odd known non-accreting magnetic white dwarfs, at least 9 have field strengths in the range $B=100-1000 \mathrm{MG}$ (Wickramasinghe \& Ferrario 2000). It was long felt that, if similar fields were present in magnetic CVs, they should retain significant optical polarization and be readily detected in CV surveys at least to the point that the cyclotron fundamental slides into the near UV $(B>300 \mathrm{MG})$. Significantly, until 1996 no polars had been found with $B>$ $100 \mathrm{MG}$, despite an overall sample similar in size to the magnetic isolated white dwarfs. The two high-field systems that have been discovered since indeed show detectable optical polarization, $0 \%-5 \%$ : AR UMa at $B=230$ MG (Schmidt 


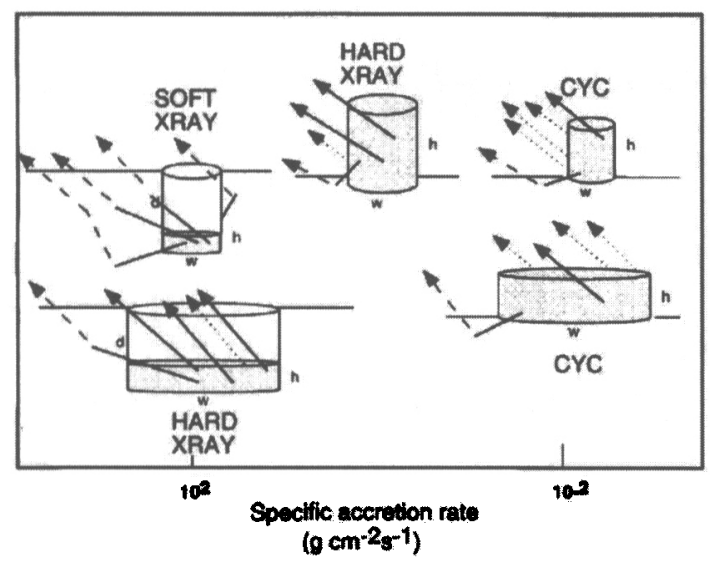

Figure 2. Shock structures on white dwarfs for a range in $\dot{m}$. The center and right columns depict cooling regions for the bremsstrahlungdominated shocks typically seen among polars. The standoff height is significant and hard X-rays and cyclotron emission can escape from the top and sides of the column. At very high accretion rates and/or high magnetic fields, the shock can become "buried" below the photosphere, as shown in the left column. Very low- $\dot{m}$ situations, where accretion energy is deposited into the atmosphere without the formation of a shock, are not represented. From Wickramasinghe \& Ferrario (2000).

et al. 1996) and V884 Her at $B=150 \mathrm{MG}$ (Schmidt et al. 2001). However, it was not adequately appreciated that the increase in cyclotron cooling, possibly coupled with "blobby" accretion, reduces the standoff height $\left(h \propto B^{-1.2}\right.$ for cyclotron-dominated shocks; Stockman 1988), buries the emission region, and again results in an enormous soft X-ray "excess" that hides them from hard X-ray surveys. For AR UMa and V884 Her, SXR/HXR $=50-1000$ (Szkody et al. 1999). The appropriate region of the $\dot{m}-B$ diagram is indicated near the upper right of Figure 1. Future searches for high-field polars should therefore emphasize soft X-ray/EUV emitters and include sensitive optical polarimetry.

\section{Polarization of Cyclotron Features}

\subsection{Theory}

The polarization characteristics of cyclotron emission are summarized in detail elsewhere (e.g., Meggitt \& Wickramasinghe 1982). Essentially, barring Faraday effects at large optical depths, circular polarization arises for sightlines that parallel the magnetic field direction, linear polarization for perspectives orthogonal to the field, and elliptical polarization for intermediate views. Relevant to the current discussion, higher harmonic cyclotron lines originate from higher-energy electrons and are more strongly beamed perpendicular to the field. Broadening of the lines is provided by Doppler motion (for the velocity component along the 

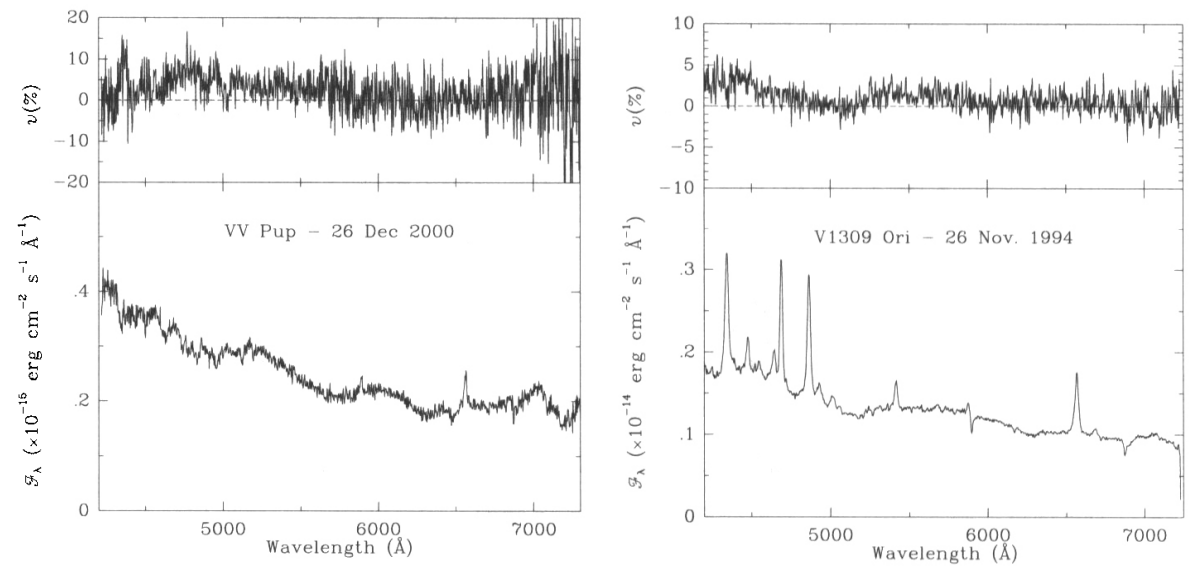

Figure 3. Spectra showing cyclotron emission lines in total flux (bottom) and circular polarization (top) for VV Pup and V1309 Ori. Magnetic field strengths are $~ 56 \mathrm{MG}$ and $61 \mathrm{MG}$, respectively.

line of sight), by the relativistic correction for electron mass, and by magnetic smearing if there exists a variation in field strength across the emission region. Finally, as the field strength increases, cyclotron lines tend to become more distinct because of both the larger wavelength separation between harmonics and the reduced shock temperature that accompanies the enhanced cyclotron cooling.

\section{2. "Classical" Polars}

The identification of cyclotron emission lines in spectroscopy of polars is a common tool for measuring the magnetic field strength at the white dwarf magnetic pole(s), and coupled with Zeeman spectroscopy of photospheric atomic lines during low accretion states, enables estimates of the field morphology over the stellar surface. As shown in Figure 3, the cyclotron features in "classical" polars are generally not well-defined. At the high shock temperatures, the lines are rendered asymmetric and for typical field strengths are broadened to the point of overlap. Doppler smearing is minimized when the accretion spot is nearest the stellar limb (B $\perp$ the line of sight), but the cyclotron light still must compete with other sources in the system, including the stellar photospheres and especially recombination emission from the funnel gas that is photoionized by the strong shock EUV. Though the net circular polarization of a polar can be large ( $v \gtrsim 20 \%$ ), harmonic overlap often causes the cyclotron lines to be even less distinct in polarization than in spectral flux, also as indicated in Figure 3.

\subsection{High-Field Systems}

The optical thickness at the cyclotron fundamental is $\Lambda \sim 10^{5}$ for typical values of $\dot{m}$, so the polarization of low-harmonic cyclotron emission is expected to be 


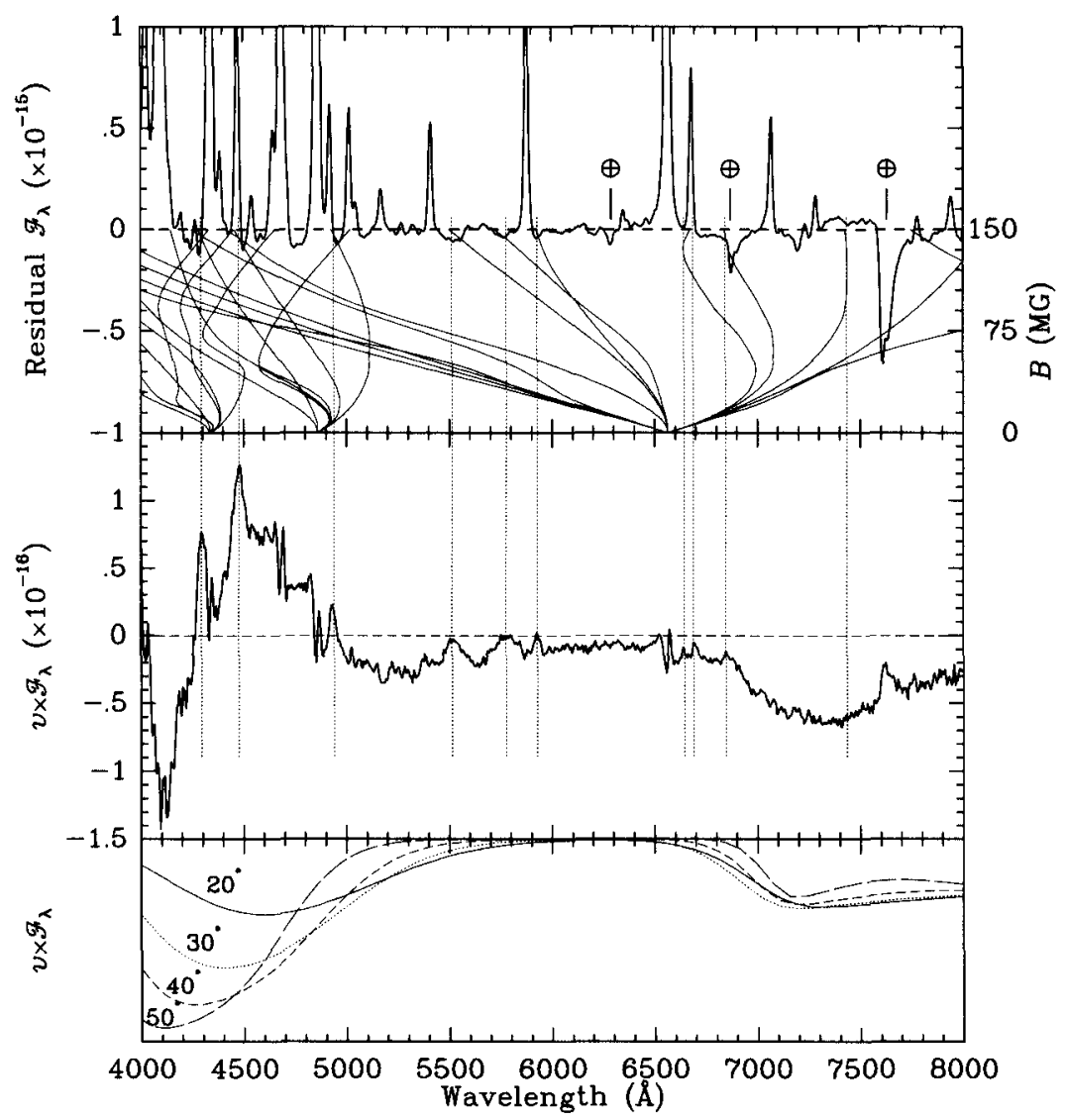

Figure 4. Top: Total flux spectrum of V884 Her after subtracting a low-order polynomial fit to the continuum. Smooth lines depict the magnetic field dependence of the Balmer series for $B=0 \rightarrow 150 \mathrm{MG}$. Middle: Phase-averaged circularly polarized flux, $v \times F_{\lambda}$. Sharp polarization reversals are evident across each major emission line, a result of Zeeman splitting within the funnel. Several positive-going polarization features - indicated by dotted lines - are accompanied by weak absorption dips in total flux and match the locations of Balmer lines for $B=150$ MG. Bottom: Model results for constant- $\Lambda, T_{e}=5 \mathrm{keV}$ shocks for various angles to the field direction. In order to fix the cyclotron fundamental at its observed wavelength around $7150 \AA$, the field strength varies over $B=115-130 \mathrm{MG}$ among the curves. From Schmidt et al. (2001). 


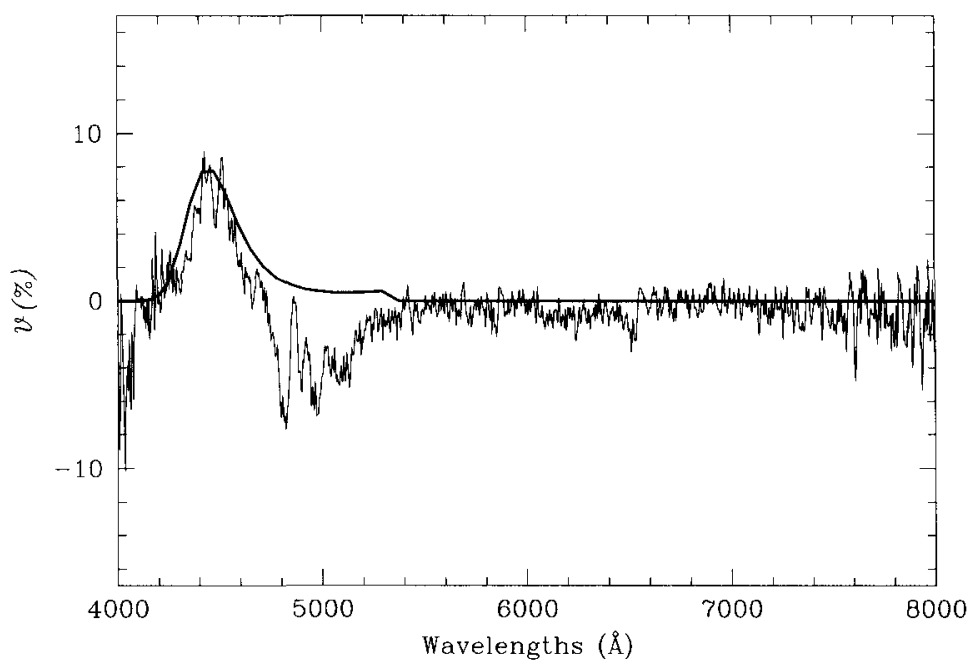

Figure 5. High-state circular polarization spectrum of AR UMa constructed by subtracting the low-state polarized flux from high-state data acquired over a full orbital cycle. The solid line is a model for emission at the $n=2$ cyclotron harmonic for $B=154 \mathrm{MG}$. This is the field strength at the weaker magnetic pole for the offset dipole model that best represents the spectrum of photospheric Zeeman absorption lines. From Ferrario et al. (2003).

small ${ }^{1}$. Furthermore, as noted in $\S 2$, the shock region can become buried so the radiation does not easily escape. Field strength measurements for high-field polars must therefore rely on the detection of Zeeman lines from the photosphere (in low accretion states) or from the light of low-density regions of the flow. Circular polarization from the latter was employed to first detect the cyclotron fundamental in a polar by Schmidt et al. (2001), as shown in a diagram reproduced here as Figure 4. V884 Her has thus far been observed only in a high accretion state, when it shows a strong blue continuum, brilliant emission-line spectrum, and net circular polarization of only $\sim 1 \%$. An array of weak absorption features in the total flux spectrum matches the Balmer spectrum of hydrogen for a magnetic field of $B=150 \mathrm{MG}$, and is interpreted as "halo" absorption from cool, partially-ionized gas overlying the accretion spot. In spectropolarimetric data, an asymmetric, negatively-polarized hump appears centered near $7150 \AA$ with amplitude $\Delta v \sim 5 \%$. This feature is weak if recognizable at all in total flux, but is at the appropriate location to be the cyclotron fundamental, produced by low optical depth $(\Lambda \sim 20)$ emission in a large area surrounding the accretion pole. A portion of a similarly-polarized $n=2$ harmonic can be discerned near the blue end of the spectrum, complicated by a confluence of positively-polarized photospheric Zeeman lines from $\mathrm{H} \beta$ and $\mathrm{H} \gamma$ (see Figure 4).

\footnotetext{
${ }^{1}$ See also the discussion by Serber elsewhere in this volume regarding this issue.
} 
Guided by this success, low-harmonic cyclotron emission has also been recognized in the other high-field polar, AR UMa. In Figure 5 we reproduce a high-state polarization spectrum from Ferrario, Wickramasinghe, \& Schmidt (2003) that shows a positively polarized hump centered near $4500 \AA$. One interpretation is that this feature is the cyclotron fundamental, which should appear around $4600 \AA$ for a field strength of $230 \mathrm{MG}$. However, Ferrario et al. choose to compare the data to a cyclotron emission model for the $n=2$ harmonic at $B=154 \mathrm{MG}$, a field strength that is also consistent with broad emission bumps in the $H S T$-UV being harmonics $n=3$ and 4 . The field strength also matches the weaker pole of a dipole whose primary pole is $235 \mathrm{MG}$ and has a longitudinal offset $\Delta r / R_{w d}=0.15$. As in V884 Her, a complex of oppositely-polarized photospheric Zeeman lines complicates the blue spectral region.

\subsection{Accretion at Very Low Mass-Transfer Rates}

New, deep optical surveys appear to be partially addressing the selection effects toward high accretion rate that were discussed in $\S 1$. The first indications of this were discoveries of two periodic variables with remarkably strong, welldefined and nearly symmetric emission humps in the Hamburg Schmidt objective prism quasar survey: HS1023+3900 (Reimers, Hagen, \& Hopp 1999) and HS0922+1333 (Reimers \& Hagen 2000). A third object with similar features is the ROSAT source 1RXS J0128-2339 (Schwope, Schwarz, \& Greiner 1999). In each case, the dramatic spectral features are found to be isolated cyclotron lines rendered particularly visible because of unusually cool emitting plasmas $(k T<2 \mathrm{keV})$ produced by very low specific accretion rates, $\dot{m} \sim 10^{-3} \mathrm{~g} \mathrm{~cm}^{-2}$ $\mathrm{s}^{-1}$. As indicated in Figure 1, such values fall in the "bombardment" regime of accretion, where no standoff shock forms, since the infalling protons can transfer their accretion energy directly to atmospheric electrons via small-angle scatterings (see Woelk \& Beuermann 1992, Rousseau et al. 1996, and references therein for a discussion of this solution.)

The Sloan Digital Sky Survey (SDSS) is also proving to be effective at finding CVs, particularly magnetic objects with cyclotron emission features that cause wide departures from the stellar locus in the 5-color photometry. Two such objects with extraordinary cyclotron lines are SDSS J155331.12+551614.5 and SDSS J132411.57+0320.5, recently reported by Szkody et al. (2003). Figure 6 presents single-phase MMT flux and polarization spectra of SDSS1553+5516 that portray the $n=3$ and 4 harmonics in a field strength of $60 \mathrm{MG}$. The enormous cyclotron lines sit atop a continuum dominated by the stellar photospheres, i.e. there is very little emission from funnel gas. Circular polarization exceeds $40 \%$ in the $n=4$ harmonic, and the large difference relative to $n=3$ indicates that the emission region is rapidly thinning in optical depth as the harmonic number increases. Particularly important is the narrow polarization structure seen within the $n=3$ harmonic. Here, the ratio $\Delta \lambda / \lambda=0.03$ for the relative width of the structure sets a firm upper limit on the plasma temperature of $T_{e}<1 \mathrm{keV}$. Over much of the orbital cycle, that harmonic displays a double-horn polarization profile characteristic of low optical-depth emission, as computed by Rousseau et al. (1996). Detailed analysis of this system, including modeling of full phase-resolved spectropolarimetry in terms of a low-temperature shock, is in preparation. 


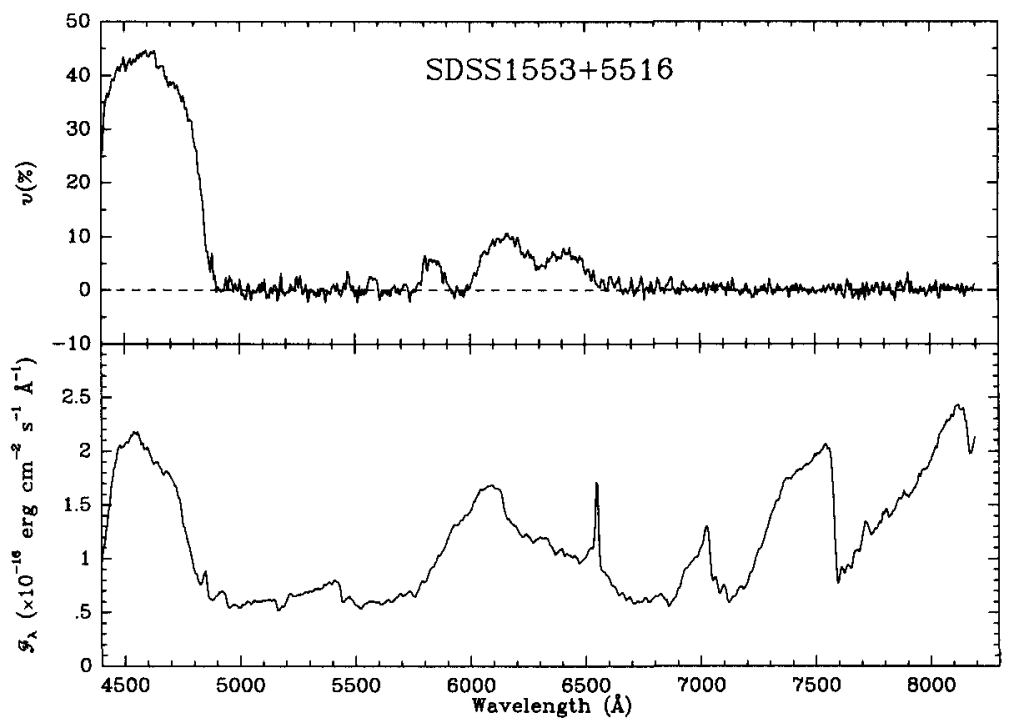

Figure 6. Total flux (bottom) and circular polarization (top) for the low- $\dot{m}$ polar SDSS1553+5516 showing very well-defined cyclotron lines $n=3(6100 \AA)$ and $n=4(4600 \AA)$ for $B=60 \mathrm{MG}$. The narrow polarization structure within the $n=3$ harmonic, $\Delta \lambda / \lambda=0.03$, implies a cool plasma, $T_{e}<1 \mathrm{keV}$. From Szkody et al. (2003).

The overall accretion rate implied by each of these low- $\dot{m}$ polars is $\dot{m}<$ $10^{-13} M_{\odot} \mathrm{yr}^{-1}$. This is about 3 orders of magnitude less than the typical rate for polars at similar orbital periods, and 2 orders of magnitude below that implied for a main-sequence secondary in contact undergoing angular momentum losses by gravitational radiation! The question clearly becomes one of the evolutionary state of the binaries and how long they remain in this very low state of accretion.

Of the 5 systems mentioned above, 1RXS J0128-2339 was presumably accreting at a more typical rate at the time of its ROSAT discovery, and the binary has recently returned to such a state (Schwope 2002). The remaining systems are not known X-ray sources (RASS upper limits for SDSS1553+5516 and SDSS1324+0320 are $0.04 \mathrm{~s}^{-1}$ and $0.02 \mathrm{~s}^{-1}$, respectively). The secondary star of SDSS1553+5516 is a good match to spectral type M5V out to the SDSS fiber spectrum limit near $9000 \AA$, and with an orbital period of $4.39 \mathrm{hr}$ it is doubtful that this system could be a post-period "bounce" system (e.g., Mennickent \& Diaz 2002; Howell, Nelson, \& Rappaport 2001). SDSS1324+0320 has a Sloan $r$ magnitude of $\sim 21$, so its near-IR spectrum is less well measured (Figure 7 ). However, the orbital period of $2.6 \mathrm{hr}$ makes it potentially more interesting from an evolutionary point of view. All of these low- $\dot{m}$ polars deserve more comprehensive study.

A spectral type of M5V for the secondary of SDSS1553+5516 permits a distance estimate, from which a maximum temperature can be derived for the white dwarf using the observed flux shortward of the $n=4$ harmonic at $4200 \AA$. The result is $T_{w d}<8000 \mathrm{~K}$ for a near-Chandrasekhar mass and $T_{w d}<5500 \mathrm{~K}$ 


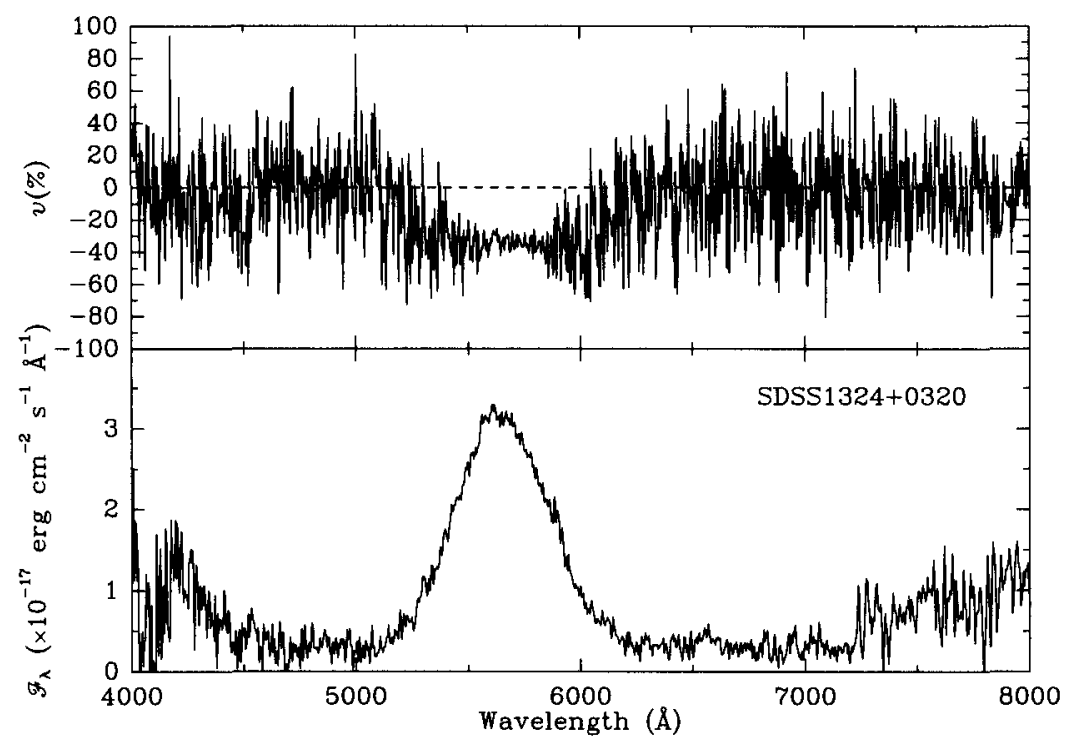

Figure 7. A single cyclotron line, interpreted as the $n=3$ harmonic in a field of $64 \mathrm{MG}$, in SDSS1324+0320. Note the weakness allowed for the white dwarf component in the blue. From Szkody et al. (2003).

for $M_{w d}=0.6 M_{\odot}$. A similar exercise for HS0922+1333 by Reimers \& Hagen (2000) obtained $T_{w d}<10,000 \mathrm{~K}$. By comparison, the coolest white dwarf in a previously-known polar (which as a CV class have the coolest primaries; Sion 1999) is MR Ser at 8500-10,500 (Schwope et al. 1993). The unusually cool white dwarfs in these CVs suggest that such low accretion states may be characteristic of the binaries, and that they may have advanced ages and/or are in unusual stages of evolution.

Acknowledgments. Continuing collaborations with L. Ferrario, P. Szkody, \& D.T. Wickramasinghe are especially appreciated. Observing assistance was provided by P. Smith. Research into magnetic white dwarfs and white dwarf accretion binaries is supported by the NSF through AST 97-30792.

\section{References}

Ferrario, L., Wickramasinghe, D.T., \& Schmidt, G. 2003, MNRAS, 338, 340

Heise, J., Brinkman, A.C., Gronenschild, E., Watson, M., King, A.R., Stella, L, \& Kieboom, K. 1985, A\&A, 148, L14

Howell, S.B., Nelson, L.A., \& Rappaport, S. 2001, ApJ, 550, 897

Lamb, D.Q., \& Masters, A.R. 1979, ApJ, 234, L117

Meggitt, S.M.A., \& Wickramasinghe, D.T. 1982, MNRAS, 198, 71

Mennickent, R.E., \& Diaz, M.P. 2002, MNRAS, 336, 767

Ramsay, G., Rosen, S.R., Mason, K.O., Cropper, M.S., \& Watson, M.G. 1993, MNRAS, 262, 993 
Reimers, D., Hagen, H.-J., \& Hopp, U. 1999, A\&A, 343, 157

Reimers, D. \& Hagen, H.-J. 2000, A\&A, 358, L45

Rousseau, T., Fischer, A., Beuermann, K., \& Woelk, U. 1996, A\&A, 310, 526

Schmidt, G.D., Ferrario, L., Wickramasinghe, D.T., \& Smith, P.S. 2001, ApJ, 553,823

Schmidt, G.D., Hoard, D.W., Szkody, P., Melia, F., Honeycutt, R.K., \& Wagner, R.M. 1999, ApJ, 525, 407

Schmidt, G.D., Szkody, P., Smith, P.S., Silber, A., Tovmassian, G., Hoard, D.W., Gänsicke, B.T., \& de Martino, D. 1996, ApJ, 473, 483

Schwope, A.D. 2002, private communication

Schwope, A.D., Beuermann, K., Jordan, S., \& Thomas, H.-C. 1993, A\&A, 278, 487

Schwope A.D., Schwarz, R., \& Greiner, J. 1999, A\&A, 348, 861

Sion, E.M. 1999, PASP, 111, 532

Stockman, H.S. 1988, in Polarized Radiation of Circumstellar Origin, eds. Coyne, G.V., S.J., et al. (Vatican: Vatican Observatory), 237

Szkody, P., Anderson, S.F., Schmidt, G. (+ 23 coauthors) 2003, ApJ, 583, 902

Szkody, P., Vennes, S., Wagner, R.W., \& Hastings, C. 1999, Annapolis Workshop on Magnetic Cataclysmic Variables, ed. C. Hoellier \& K. Mukai, ASP Conf. Ser., 157, 195

Warner, B. 1995 Cataclysmic Variable Stars, (Cambridge: Cambridge Univ. Press), 343

Wickramasinghe, D.T., \& Ferrario, L. 2000, PASP, 112, 873

Woelk, U., \& Beuermann, K. 1992, A\&A, 256, 498 